Gratings in indium oxide film overlayers on ion-exchanged waveguides by excimer laser micromachining

\author{
S. Pissadakis*, L. Reekie ${ }^{\dagger}$, M.N. Zervas, J.S. Wilkinson \\ Optoelectronics Research Centre, University of Southampton, \\ SO17 1BJ, Southampton, United Kingdom
}

\title{
G. Kiriakidis
}

FO.R.T.H-IESL, Materials group, P.O. Box 1527,

Heraklion 71 110, Crete, Greece

\begin{abstract}
Relief Bragg gratings were imprinted by $248 \mathrm{~nm}$ interferometric excimer laser ablation on potassium ion-exchanged channel waveguides in BK-7 glass overlaid with a thin high-index $\operatorname{InO}_{x}$ film. Using five pulses of energy density $60 \mathrm{~mJ} / \mathrm{cm}^{2}$, a spectral transmittance notch of depth $66 \%$ and $\Delta \lambda_{\text {FWнM }}<0.1 \mathrm{~nm}$ was obtained at $1547 \mathrm{~nm}$ in the TE polarisation for a waveguide having a nominal width of $8 \mu \mathrm{m}$ and a $135 \mathrm{~nm}$ thick $\mathrm{InO}_{\mathrm{x}}$ overlayer. In waveguides coated with $100 \mathrm{~nm}$ $\mathrm{InO}_{\mathrm{x}}$, with widths increasing from $3 \mu \mathrm{m}$ to $8 \mu \mathrm{m}$, the reflection wavelength shifted by $0.12 \mathrm{~nm} / \mu \mathrm{m}$ and the reflectivity increased monotonically.
\end{abstract}

\footnotetext{
* e-mail: sp1@orc.soton.ac.uk

$†$ Current address: JDS Uniphase Pty. Ltd., 4 Byfield Street, North Ryde, NSW 2113, Australia
} 
Waveguide gratings are potentially important components in integrated optical circuits, providing signal filtering and routing with high extinction ratio, while allowing dense integration, robustness and stability. Bragg gratings were realised in planar waveguides in the $1970{ }^{1} \mathrm{~s}^{1}$ and have since been used extensively in line-narrowing semiconductor lasers. Recently, research into photosensitive $\mathrm{e}^{2,3}$ and relief ${ }^{4,5}$ gratings has intensified due to renewed interest in integrated optical devices using alternative materials for telecommunications. Relief gratings may readily be applied to the majority of waveguide materials without requiring special photosensitive materials. This increases the design flexibility for the realisation of high performance waveguide grating devices based on such alternative materials and optical configurations. Additionally, the application of excimer lasers to the fine machining of optical materials has recently been intensively investigated. Interferometric laser ablation has the potential for realising strong gratings in compact waveguide devices, with the advantages of flexibility of wavelength selection and minimisation of the number of process steps. We have recently demonstrated such reflection gratings at wavelengths near $1300 \mathrm{~nm}$, directly written in thallium ion-exchanged glass waveguides by interferometric laser ablation at $193 \mathrm{~nm}^{6}$. However, the exposure caused substantial damage to the waveguides, resulting in significant broadband losses. Nevertheless, interferometric excimer laser ablation is a flexible, simple and reliable relief grating patterning method, which has been used in the past for the imprinting of high-quality sub-micron relief gratings in thin oxide films ${ }^{7}$. Bragg relief gratings have also been written by e-beam lithography and etching of titanium diffused waveguides in lithium niobate overlaid with a thin silicon film to enhance modal overlap with the grating ${ }^{8}$. 
In this Letter we present waveguide reflection sub-micron relief gratings on multilayer waveguides operating near $1550 \mathrm{~nm}$, fabricated by interferometric excimer laser ablation at $248 \mathrm{~nm}$. The devices consisted of monomode potassium ion-exchanged channel waveguides in BK-7 glass overlaid for part of their length with a thin sputtered indium oxide $\left(\mathrm{InO}_{x}\right)$ film in which the gratings were written, as shown in Figure 1. The refractive index of the deposited layer $(n \approx 1.7-1.8)$ is significantly higher than the maximum refractive index of the diffused channel waveguide, causing the optical fields to be drawn up to the surface and interact strongly with the film 9 . The thickness of the overlayer is chosen so that it is below cut-off and, therefore, it does not itself form a waveguide in the wavelength region of interest. Indium oxide was chosen as it may be machined using low energy densities, avoiding damage to the underlying ion-exchanged region. Light from a single-mode telecommunications fibre, butt-coupled to the polished endface, excites the mode of the uncoated input section of the waveguide (A) and crosses the transition into the coated region (B). The modal intensity distribution in the coated region is drawn up to the surface by the high index film, as shown schematically in Figure 1, resulting in larger overlap and increased sensitivity to surface perturbations. The thickness of the overlayer is ideally chosen to be small enough so that the coated waveguide is monomode and the scattering losses at the transitions are low. If a relief or photorefractive grating is recorded in the thin film overlayer (C) the field enhancement at the surface provides for strong interaction of the guided field with the grating.

Waveguides were fabricated by ion exchange in two BK7 glass substrates in molten potassium nitrate through aluminium mask openings ranging from $3 \mu \mathrm{m}$ to $8 \mu \mathrm{m}$, at $400^{\circ} \mathrm{C}$ for 11 hours ${ }^{10}$. 
Polycrystalline indium oxide thin films one of thickness $100 \mathrm{~nm}$ and one of thickness $135 \mathrm{~nm}$ were sputtered on two waveguide chips using dc-magnetron sputtering in a $100 \% \mathrm{O}_{2}$ atmosphere to cover a $25 \mathrm{~mm}$ length in the centre of the $40 \mathrm{~mm}$ long waveguides. The overlaid waveguides were exposed to a high-contrast UV fringe pattern with period of $514.3 \mathrm{~nm}$ using the three-mirror interferometer described previously ${ }^{6}$. The spatial distribution of the laser output was homogenised during recording using a rotating fused silica plate. Gratings of length $16 \mathrm{~mm}$ were produced on each set of waveguides by exposure to an average pulse energy density of 45 $60 \mathrm{~mJ} / \mathrm{cm}^{2}$ using between 5 and 100 pulses. An SEM micrograph of a grating ablated in a $100 \mathrm{~nm}$ thick $\mathrm{InO}_{\mathrm{x}}$ film using 20 pulses of energy density $45 \mathrm{~mJ} / \mathrm{cm}^{2}$ is shown in Fig. 2.

Waveguide transmission spectra were obtained before $\mathrm{InO}_{\mathrm{x}}$ deposition and after grating micromachining. Broadband ASE from an erbium-doped fibre amplifier was coupled into each waveguide in turn using a monomode fibre. The waveguide output was collected by two x10 microscope objective lenses and an intermediate IR polariser and launched into a multimode fibre coupled to an optical spectrum analyser. The polarised transmission spectra for the waveguides were found by normalising these spectra to that obtained with the monomode fibre butted directly to the multimode fibre. Reflection spectra were obtained using a fibre coupler at the input. The transmission losses of the waveguides before deposition of the $\mathrm{InO}_{\mathrm{x}}$ films, including input fibre coupling losses, were less than $3 \mathrm{~dB}$ for both polarisations.

The transmission and reflection spectra for the TE polarisation of an $8 \mu \mathrm{m}$ wide channel waveguide overlaid with a $135 \mathrm{~nm}$ thick $\mathrm{InO}_{\mathrm{x}}$ film supporting a grating ablated using 5 pulses of 
energy density $60 \mathrm{~mJ} / \mathrm{cm}^{2}$ are shown in Fig. 3. The average grating depth was estimated to be approximately $20 \mathrm{~nm}$ by atomic force microscope measurements. The transmission spectrum shows a broadband loss of about $4 \mathrm{~dB}$, mainly due to fibre-waveguide coupling loss, including the transition losses between the coated and uncoated waveguide regions and propagation losses in the overlayer. The grating transmission showed a clear notch at $1546.7 \mathrm{~nm}$ with a depth of approximately $4.7 \mathrm{~dB}(66 \%)$ and a bandwidth at full-width half-maximum power $\left(\Delta \lambda_{\text {FWHM }}\right)$ of $0.08 \mathrm{~nm}$. At wavelengths shorter than the Bragg wavelength the transmission spectrum shows an increased broadband loss, which is attributed to coupling to radiation modes. The insertion loss of the waveguide overlayer clearly remained low even after UV exposure of the film. Exposures with higher energy densities or a larger numbers of pulses resulted in significantly greater absorption loss and weaker grating strengths. Annealing of the waveguide chip up to $250^{\circ} \mathrm{C}$ for two hours in an oxygen atmosphere reduced the loss further by almost $1 \mathrm{~dB}$. The reflection spectrum shows that reflected power is strongly coupled into the backward-travelling waveguide mode at $1546.7 \mathrm{~nm}$. However, the grating strength in reflection is reduced to approximately $38 \%$ due to the additional background losses. No detectable grating response was observed for the TM polarisation in reflection or transmission due to the much lower overlap of the guided mode with the grating in this polarisation.

Figure 4(a) shows the reflection spectra for waveguides with ion-exchange mask widths from $3 \mu \mathrm{m}$ to $8 \mu \mathrm{m}$, coated with $100 \mathrm{~nm} \mathrm{InO}_{\mathrm{x}}$ and exposed to 20 pulses of energy density $60 \mathrm{~mJ} / \mathrm{cm}^{2}$. Figure 4(b) summarises these data by showing the reflection wavelength and peak reflectivity plotted against ion-exchange mask width. There is a near-linear dependence of the Bragg grating 
wavelength on the waveguide width, exhibiting a slope of $0.12 \mathrm{~nm} /$ micron width change over this range. The grating strength in reflection increased from $3.0 \%$ and $14.8 \%$ for the same range of channels. The increase of grating reflectivity with the waveguide width is attributed to the greater confinement of the guiding mode closer to the surface of the waveguide channel, resulting in stronger interaction with the grating corrugation. Grating strengths up to $20 \mathrm{~dB}$, accompanied by higher transmission losses, have also been observed in $\mathrm{Ta}_{2} \mathrm{O}_{5}$ overlaid devices and further details and comparative data will be presented elsewhere.

In conclusion, relief Bragg reflection gratings have been micromachined by $248 \mathrm{~nm}$ interferometric excimer laser ablation on potassium ion-exchanged channel waveguides in BK-7 glass overlaid with a thin high-index $\mathrm{InO}_{\mathrm{x}}$ film. Using five pulses of energy density $60 \mathrm{~mJ} / \mathrm{cm}^{2}$, a narrowband spectral transmittance notch of at least $66 \%$ and $\Delta \lambda_{\mathrm{FWHM}}<0.1 \mathrm{~nm}$ was obtained at $1547 \mathrm{~nm}$ in the TE polarisation for a waveguide having a nominal width of $8 \mu \mathrm{m}$ and a $135 \mathrm{~nm}$ thick $\mathrm{InO}_{\mathrm{x}}$ overlayer. In waveguides coated with $100 \mathrm{~nm} \mathrm{InO}$, with widths increasing from $3 \mu \mathrm{m}$ to $8 \mu \mathrm{m}$, the reflection wavelength shifted by $0.12 \mathrm{~nm} / \mu \mathrm{m}$ and the reflectivity increased monotonically. Low-loss, polarisation insensitive devices based on high-index overlaid waveguide gratings may be used for gain flattening or for wavelength add-drop applications. Further optimisation of the device is directed towards the reduction of the UV-induced losses, and control of polarisation sensitivity through optimisation of overlayer film thickness. 


\section{Figure captions}

Fig. 1. Schematic representation of a high-index overlayer grating on an ion-exchanged waveguide. A: propagating mode in uncoated region. B: mode enhancement in the highindex overlaid region. $\mathrm{C}$ : interaction of the enhanced mode with the high-index grating corrugation.

Fig. 2. Grating ablated in a $100 \mathrm{~nm}$ thick $\mathrm{InO}_{\mathrm{x}}$ film using 20 pulses of $45 \mathrm{~mJ} / \mathrm{cm}^{2}$ energy density.

Fig 3. Transmission (thick line) and reflection (thin line) spectra for an $8 \mu \mathrm{m}$ wide channel waveguide overlaid with $135 \mathrm{~nm}$ thick $\mathrm{InO}_{\mathrm{x}}$ film.

Fig. 4. (a) Grating reflection spectra and (b) Peak reflectivity (circles) and Bragg wavelength (squares) vs. waveguide width, for the TE polarisation for waveguides overlaid with a $100 \mathrm{~nm}$ thick $\mathrm{InO}_{\mathrm{x}}$ film exposed to 20 pulses of energy density $60 \mathrm{~mJ} / \mathrm{cm}^{2}$. 


\section{References}

' D. C. Flanders, H. Kogelnik, R.V. Schmidt, C.V. Shank, Appl. Phys. Lett. 24, 194, (1974)

${ }^{2}$ D.F. Geragthy, D. Provenzano, W.K. Marshall, S. Honkanen, A. Yariv, N. Peyghambarian, Electron. Lett. 35, 585 (1999)

${ }^{3}$ M. Ibsen, J. Hubner, J.E. Pedersen, R. Kromann, L.-U.A. Andersen, M. Kristensen, Electron. Lett. 32, 2233 (1996)

${ }^{4}$ J.E Roman, K.A. Winick, Appl. Phys. Lett. 61, 2744 (1992)

${ }^{5}$ R. Adar, C.H. Henry, R.C. Kistler, R.F. Kazarinov, Appl. Phys. Lett. 60, 1779 (1992)

${ }^{6}$ S. Pissadakis, L. Reekie, M. Hempstead, M.N. Zervas, J.S. Wilkinson, Appl. Surf. Sci. 153, 200 (2000)

${ }^{7}$ S. Pissadakis, L. Reekie, J.S. Wilkinson, G. Kiriakidis, Proc. CLEO-Europe, Nice, France, CWF50 (2000)

${ }^{8}$ C.P. Hussel, R.V. Ramaswamy, IEEE Phot. Technol. Lett. 9, 636 (1997)

${ }^{9}$ G.R.Quigley, R.D.Harris, J.S.Wilkinson, Appl.Opt. 38, 6036 (1999)

${ }^{10}$ T.Feuchter, E.K.Mwarania, J.Wang, L.Reekie, J.S.Wilkinson, Phot. Technol. Lett. 4, 542 (1992) 


$$
1
$$




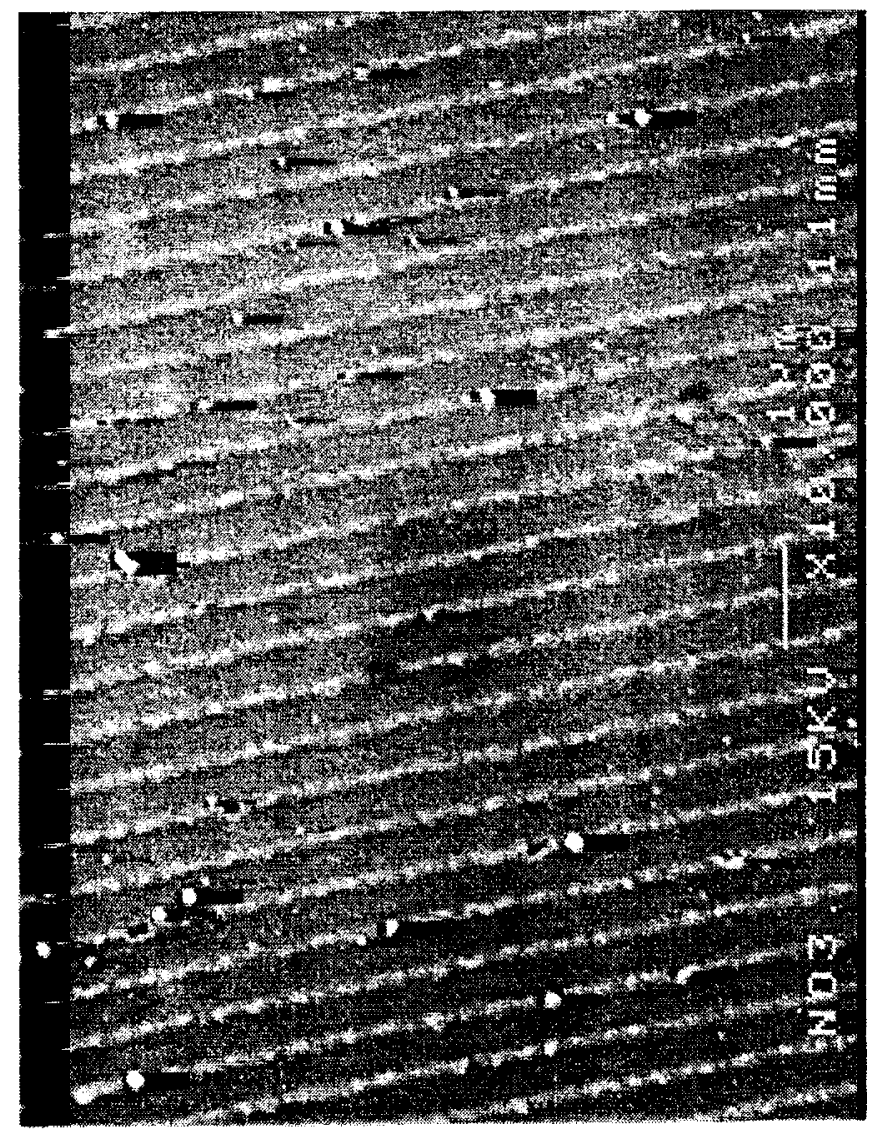


$M$

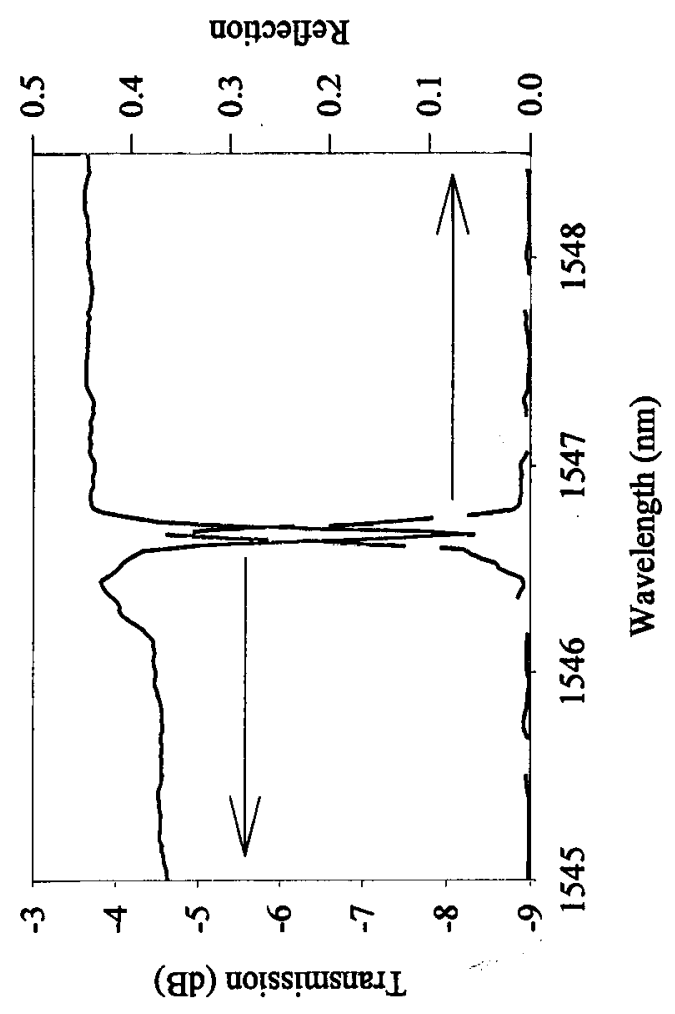



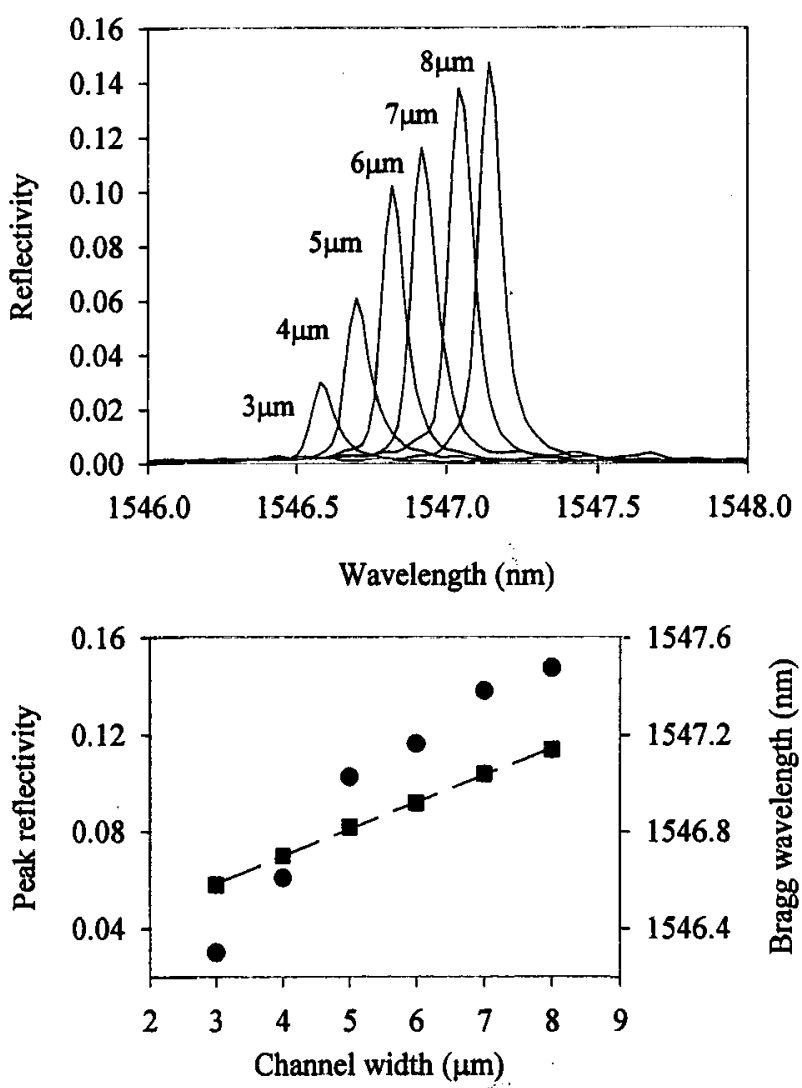

Fig. 4 